\title{
Effects of Diglycerol Tetranitrate Plasticizing Different Polymers on Hygroscopicity of Ammonium Nitrate
}

\author{
Xiaolan Song ${ }^{1, *}$, Chenli Liu ${ }^{1}$, Yi Wang ${ }^{2}$, Zhipeng Cheng ${ }^{3}$ and Fengsheng $\mathrm{Li}^{4}$ \\ ${ }^{1}$ School of Environment and Safety Engineering, North University of China, Taiyuan 030051, China \\ ${ }^{2}$ School of Materials Science and Engineering, North University of China, Taiyuan 030051, China \\ ${ }^{3}$ School of Chemistry \& Chemical Engineering, Huaiyin Normal University, Huaian 223300, China \\ ${ }^{4}$ School of Chemical Engineering, Nanjing University of Science and Technology, Nanjing 210094, China \\ Email: songxiaolan00@126.com
}

\begin{abstract}
A large number of studies have shown that there are two main methods for the hygroscopicity of ammonium nitrate (AN). One is spheroidization and the other is coating modification. In this study, numerical simulation is first performed and then spherical AN is prepared and modified by coating. In theoretical simulation section, the binding energy of Polystyrene (PS), Polymethylmethacrylate (PMMA), Polyvinyl Chloride, and Nitrocellulose (NC) and explosive AN were simulated using Materials Studio software. In the experimental part, ultrafine AN is obtained by mechanical ball milling, then the surface of the refined AN is coated with a certain mass fraction of these four kinds polymerA certain amount of Diglycerol Tetranitrate (DGTN) is added during the coating to increase the plasticity and film formation of the polymer. The raw material AN and the four polymer-coated AN samples were subjected to microscopic morphology and particle size analysis (SEM), thermal decomposition characteristics analysis (DSC), X-ray powder diffraction analysis (XRD), infrared spectroscopy (IR), and moisture absorption test. The results show that the binding energy between the four materials and $\mathrm{AN}$ is in order: $\mathrm{PMMA}>\mathrm{PVC}>\mathrm{NC}>\mathrm{PS}$. The refined $\mathrm{AN}$ particle diameter obtained by mechanical ball milling is lower than 5 micrometers, which is ellipsoidal and does not change the crystal form of AN. The four coating materials are effectively coated with AN's surface, and the AN is not changed before and after coating about crystal structure. The effect of the four coating materials on the AN hygroscopicity is: $\mathrm{NC}>\mathrm{PVC}>\mathrm{PMMA}>\mathrm{PS}$. The AN moisture absorption rate after coating with PS, PMMA, PVC, and NC decreased by 11.11\%, 21.48\%, 45.94\%, and 46.91\%, respectively, compared with the moisture absorption rate of the raw material AN.
\end{abstract}

Keywords: AN, DGTN, polymers, surface coating, hygroscopicity

\section{Introduction}

Ammonium nitrate (AN) is an important part of industrial explosives and solid propellants ${ }^{[1]}$. AN is also a kind of agricultural fertilizer ${ }^{[2]}$. The price of AN is relatively low and easy to obtain. Compared with Ammonium Perchlorate (AP), it does not contain halogen. AN does not produce corrosive gases such as $\mathrm{HCl}$. Therefore, it has no characteristic signal and can reduce the pollution to the atmosphere $\mathrm{e}^{[3,4]}$. When AN absorbs moisture, it can accelerate the crystal transformation of AN and reduce its crystal transition point ${ }^{[5]}$. For example, when the water content in AN increases from $0.05 \%$ to $3.4 \%$, the transition temperature of IV $\rightarrow$ III decreases from $48{ }^{\circ} \mathrm{C}$ to $37^{\circ} \mathrm{C}$. When the water content increases from $0.12 \%$ to $3.4 \%$, the transition temperature of $\mathrm{III} \rightarrow \mathrm{II}$ decreases from $125^{\circ} \mathrm{C}$ to $120^{\circ} \mathrm{C}^{[6,7]}$. When $\mathrm{AN}$ is used in propellants, the hygroscopicity of AN seriously affects the combustion performance and storage stability of the propellant ${ }^{[8,9]}$. What's more, when $\mathrm{AN}$ is used as a fertilizer, the moisture absorption will cause agglomeration and affects its mechanized fertilization ${ }^{[10]}$. So, how to reduce the moisture absorption of AN is the key to full and effective use of AN.

The root cause of AN's moisture absorption is that its particle surface is a polar high energy surface and its crystal surface structure is a porous void structure. Since the surface of the AN is a polar high energy surface, the AN particles strongly absorb moisture in the air. And the AN particles change from a high-energy surface to a low-energy surface after adsorbing water molecules by electrostatic interaction and hydrogen bonding with water molecules ${ }^{[11,12]}$. When the adsorbed moisture reaches a certain level, the 
surface of the AN particles dissolves and forms a film of a saturated solution. According to Raoul's law, when the partial pressure $\mathrm{P}$ of the water vapor in the air is greater than the partial pressure $\mathrm{P}_{\mathrm{h}}$ of the water vapor on the saturated ammonium nitrate solution on the surface of the particle, the ammonium nitrate particles absorb moisture from the air ${ }^{[13]}$. The AN particles are completely dissolved due to the high solubility of the AN particles. A liquid bridge is formed between the liquid films of the dissolved particles. The AN in the liquid crystal crystallizes will form a salt bridge to agglomerate the AN particles when the temperature drops. Secondly, the AN particles have a large porosity which causes the AN particles to have a great adsorption effect on water molecules. Therefore, the key to reduce the hygroscopicity of AN is to improve the surface structure, reduce the surface energy and increase the water repellency ${ }^{[14,15]}$. There have been a lot of researches on the anti-hygroscopicity of AN at home and abroad, mainly spheroidization and coating a layer of hydrophobic material.

At present, there are several methods to prevent AN's moisture absorption. The first method is to add an inorganic salt additive to the AN particles. The inorganic salt additives added to the AN particles mainly contain nitrates, phosphates, sulfates and their composite systems. The inorganic salt additive is added to the AN particles by adding these additives to the solution or melt of the AN for granulation in the production process of AN. This method reduces the hygroscopicity of AN by combining inorganic salt particles with free water in AN or changing the crystal habit or reducing the void ratio of $\mathrm{AN}^{[16]}$. The second method is to coat the surface of the AN particles with organic hydrophobes materials. Commonly used hydrophobic substances are mainly mineral oil, paraffin wax, halogenated silane, octadecane diamide, sorbitol tetranitrate and rosin ${ }^{[17]}$. The hydrophobic material is added to the molten AN or the solution is sprayed onto the surface of the AN particles, which can form a hydrophobic film on the surface of the AN. By this method, the surface energy of the AN can be lowered, the water repellency can be improved, and the moisture absorption can be prevented ${ }^{[18]}$. The third method is to surface the AN with a surfactant. Because the surfactant has an amphiphilic structure, its hydrophilic group combines with the surface of the AN particle by electrostatic interaction and hydrogen bonding. And the lipophilic group forms a hydrophobic film on the outer side of the AN. This method reduces the original surface energy of the AN particles, effectively reduces the direct contact between the water molecules and the AN particles, and reduces the hygroscopicity of the AN. This is the main method currently used to reduce the hygroscopicity of ammonium nitrate. In this method, the surfactant is made into a solution, sprayed onto the surface of the AN particles, or the surfactant is added to $90 \%$ to $95 \%$ of the AN solution for vacuum crystallization to obtain an anti-hygroscopic $\mathrm{AN}^{[19,20]}$. The fourth method is to coat the AN with a polymer. Spraying a polymer such as nitrocellulose, polyethylene, polyester, etc. On the surface of the AN particles in the form of a solution, a thin, dense, elastic and tough hydrophobic film can be formed on the surface of the AN particles. This method can reduce the surface energy of the AN and prevent the direct contact between the surface of the AN particles and the water vapor molecules, thereby reducing the hygroscopicity of the $\mathrm{AN}^{[21]}$.

In this paper, PS, PMMA, PVCand NC are selected as coating materials. The binding energy of four cladding materials to the main explosive AN was calculated by Materials Studio software. Analyze the tightness of the combination of four materials with AN by analyzing the numerical of binding energy. The AN material was refined by mechanical ball milling during the experiment. Mechanical ball milling is a green safe refinement method ${ }^{[22]}$. A small amount of toluene solvent is added to the ball mill tank together with the ball mill beads and the AN raw material. Then the AN particles and the ball mill beads are thoroughly mixed by high-speed rotation. Through the extrusion and grinding of the ball beads, the AN materials become fine and rounded in a short time. Then coating the surface of the refined AN with a surfactant octadecylamine ${ }^{[23,24]}$. TheDGTNis mixed with each coating material and coated on the AN surface. DGTN is a non-volatile energetic plasticizer with higher energy and lower sensitivity. What's more, its operational safety is also relatively high ${ }^{[25]}$. PS, PMMA, PVC and NC all have certain solvent resistance or water resistance. They can form a film-forming hydrophobic film on the surface of the explosive to improve the hygroscopicity of the explosive ${ }^{[26,27]}$. Finally, the effects of four polymers on the hygroscopicity of AN before and after coating were tested which provided theoretical support for the storage and use of $\mathrm{AN}^{[28]}$. 


\section{$2 \quad$ Experimental Method}

\subsection{Materials}

Ammonium nitrate (C.P.), Jinan Century Tongda Chemical Co., Ltd.; DGTN raw materials and NC raw materials, North University of China; PS raw materials, Guangdong Dongguan Shunjie Plastic Co., Ltd.; PMMA raw materials, Suzhou Yuchenlong Engineering Plastics Co., Ltd.; PVC raw materials, Shandong Shouguangyu Waterproof Material Co., Ltd.; Toluene, Guangshen Chemical Co., Ltd.; Zirconium beads $\left(\varphi=5 \mathrm{~mm}, \mathrm{ZrO}_{2}\right.$ doped with $\left.2 \sim 10 \mathrm{wt} \% \mathrm{Y}_{2} \mathrm{O}_{3}\right)$, Shandong Zibo Yubang Industrial Ceramics Co., Ltd.

\subsection{Samples Preparation}

The refinement process: 1) The raw material AN, zirconium beads and toluene are simultaneously placed in a ceramic ball mill jar and stirred uniformly; 2) The ball mill tank is fixed in a Miqi planetary ball mill (YXQM-1L), and the revolution speed is set to $350 \mathrm{r} / \mathrm{min}$, after starting grinding for $6 \mathrm{~h}$, the material was taken out; 3) The zirconium ball was separated from the material, and after suction filtration and freeze-drying, an ellipsoidal refinement AN having a particle diameter of $5 \mu \mathrm{m}$ was obtained.

Coating process: 1) Dissolve $0.7 \mathrm{~g}$ octadecylamine in chloroform solution and add $70 \mathrm{~g}$ refined AN to the above solution. Stir evenly in the ultrasonic oscillator until it is muddyand dry for use; 2) $20 \mathrm{~g}$ the AN of octadecylamine is placed in four mortars in an average. And 0.1g PS, PMMA, PVC and NC were dissolved in $6 \mathrm{ml}$ chloroform solution, chloroform solution, tetrahydrofuran solution and acetone solution, respectively. Then add 0.1g DGTN to the above four solutions, respectively. Finally, the four mixed liquids are separately added to the four mortars equipped with $\mathrm{AN}$, stirred uniformly, and dried to prepare samples.

\subsection{Characterization}

The microscopic morphology analysis is performed by field emission scanning electron microscopy SEM (JEOL jsm-7500). The thermal analysis is performed by DSM-60 differential scanning calorimeter from Shimadzu Corporation, Japan. The heating rate is $20{ }^{\circ} \mathrm{C} / \mathrm{min}$, air atmosphere, ceramic crucible, the crucible was closed during the test. Analyze the crystalline phase of different samples is carried out by German Burker Advance D8 X-ray powder diffractometer X-Ray Diffraction, XRD. Molecular structure analysis uses American Thermo Scientific Nicolet 6700 infrared spectrometer IR.

Hygroscopicity test: The samples are placed at $25^{\circ} \mathrm{C}$, the relative humidity is $75 \%$. The hygroscopicity test is performed and the weight is recorded every hour. The average is recorded twice per record, the data of the first 10 hours is recorded, and the 24th hour iss recorded.

\section{$3 \quad$ Results and Discussion}

\subsection{Binding Energy between AN and Cladding Materials}

Table 1. Unit cell parameters, optimization values and errors of AN crystals.

\begin{tabular}{llllllll}
\hline \multicolumn{2}{c}{ Crystal } & $\mathbf{a} / \AA$ & $\mathbf{b} / \AA$ & $\mathbf{c} / \AA$ & $\boldsymbol{\alpha} /{ }^{\circ}$ & $\boldsymbol{\beta} /{ }^{\circ}$ & $\boldsymbol{\gamma}^{\circ}{ }^{\circ}$ \\
\hline \multirow{4}{*}{$\mathrm{AN} \quad$ Experimental value } & 6.414 & 6.581 & 10.412 & 90 & 90 & 90 \\
& Universal & 5.875 & 6.793 & 10.054 & 90 & 90 & 90 \\
& Error $/ \%$ & 8.41 & -3.22 & 3.44 & 0 & 0 & 0 \\
\hline
\end{tabular}

Firstly we obtained the AN cell structure from the Cambridge Crystallographic Data Centre (CCDC). The unit cell structure of AN is shown in Figure 1 below. Then select the Universal Field as the initial force field for the analog AN crystal[ ${ }^{[29]}$. The AN unit cell is geometrically optimized, and the optimized unit cell parameters and their errors are shown in Table 1. 


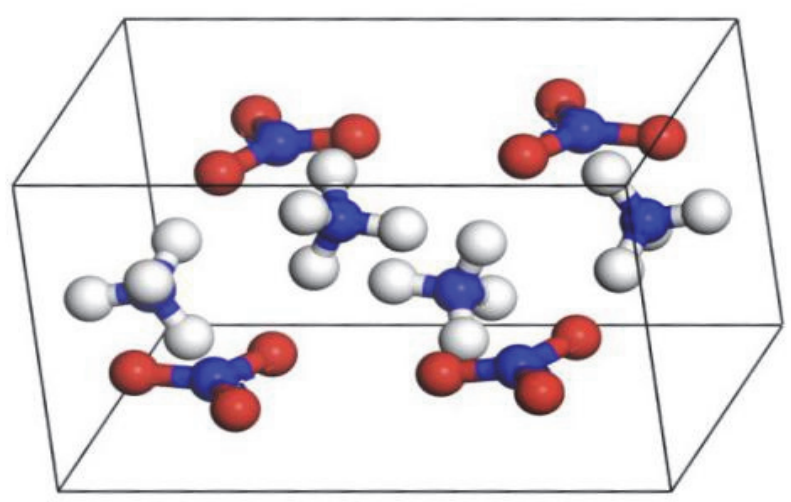

Figure 1. The unit cell structure of AN.

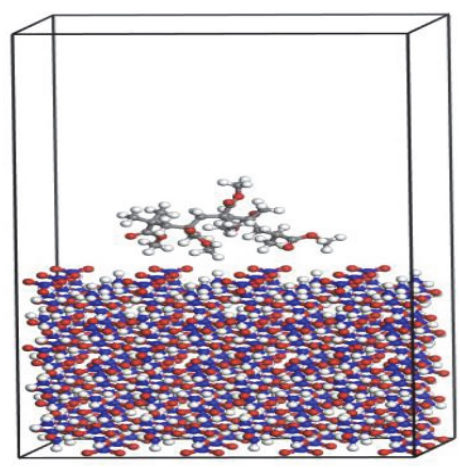

(a)

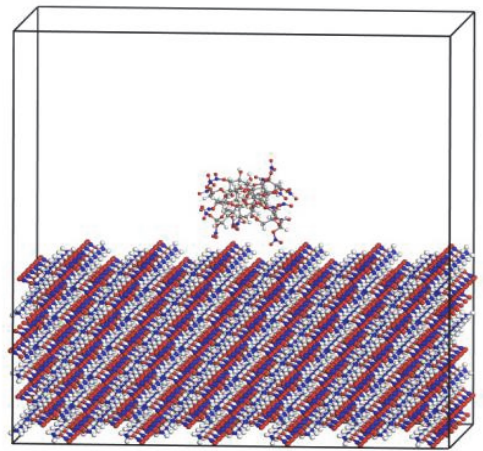

(c)

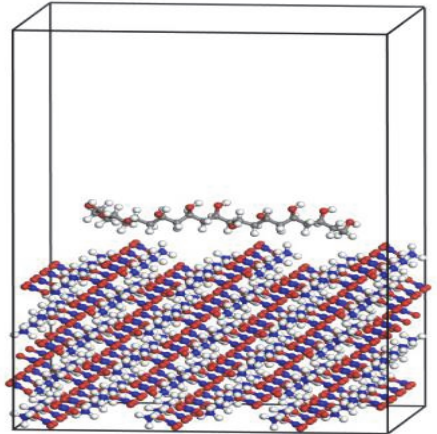

(b)

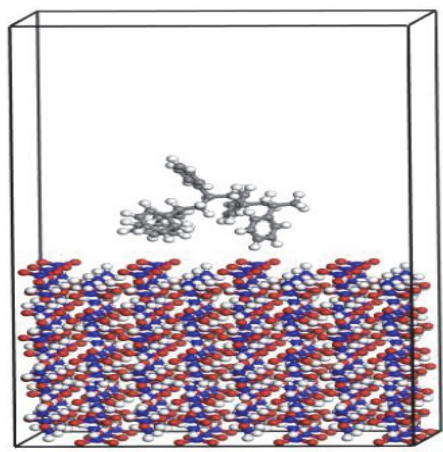

(d)

Figure 2. The structural models of the four polymers and AN.

This simulation uses additives with a mass fraction of $3 \%$ and crystals of $97 \%$.Cutting the unit cell to obtain the AN(1 $\left.\begin{array}{lll}1 & 1\end{array}\right)$ crystal plane and expanding it into a super unit cell. Then we optimize the supercell structure. Draw the initial structure of the additive molecule and construct polymer molecules to geometrically optimize the polymer molecules. The optimized polymer molecules are then placed above the crystal plane along the $\mathrm{Z}$ axis and held a certain distance. Perform geometric optimization on the initial structure of the model. The structural models of the four polymers and AN are shown in Figure 2. Figure (a) shows the structure of AN and PMMA. Figure (b) shows the structure of AN and PVC. Figure (c) shows the structure of AN and NC. Figure (d) shows the structure of AN and PS. Then we calculate the van der Waals interaction and electrostatic interaction by using Atom Based and Ewald methods. The binding energy between the crystal face and the polymer molecule (Ebind) is calculated according to the following formula ${ }^{[30,31]}$ : 


$$
E_{\text {bind }}=E_{\text {tot }}-\left(E_{c r y}+E_{\text {add }}\right)
$$

where $\mathrm{E}_{\text {tot, }}, \mathrm{E}_{\text {cry }}$ and $\mathrm{E}_{\text {add }}$ represent the total energy of the model, the energy of the crystal face layer and the energy of the polymer molecule.

The binding energy between the additives and the crystal face are shown in the Table 2. For the bonding system of AN(101) crystal plane, the order of the absolute value of the binding energy between the additive and the crystal plane is: PMMA $>$ PVA $>\mathrm{NC}>\mathrm{PS}$. The results show that the order of interaction between the additives and the crystal face is: $\mathrm{PMMA}>\mathrm{PVA}>\mathrm{NC}>\mathrm{PS}$.

Table 2 . The binding energy between the additives and the crystal face $\left(\mathrm{kcal} \cdot \mathrm{mol}^{-1}\right)$.

\begin{tabular}{lllll}
\hline Additives & PS & PMMA & PVA & NC \\
\hline AN $\left(\begin{array}{lll}1 & 0 & 1\end{array}\right)$ & -22.32 & -53.38 & -42.15 & -34.12 \\
\hline
\end{tabular}

\subsection{SEM Test Results of AN Samples before and after Coating}

SEM tests were performed on different samples and the results are shown in Figure 3. Figure (a) is the image of the raw material AN after ball milling. Figures (b), (c), (d), and (e) are AN samples coated with PS, PMMA, PVC, and NC, respectively. It can be seen in the figures that the surface of the AN before coating is smooth and loose, and the surface of the samples after coating is rough and dense. Moreover, it can be seen that there is a film on the surface of the coated explosive. SEM test results show that PS, PMMA, PVC and NC are effectively coated with AN

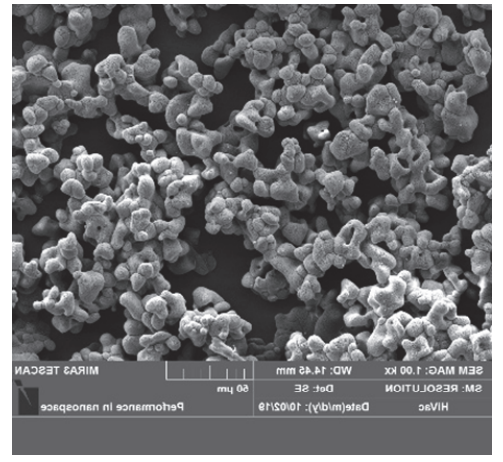

(a)

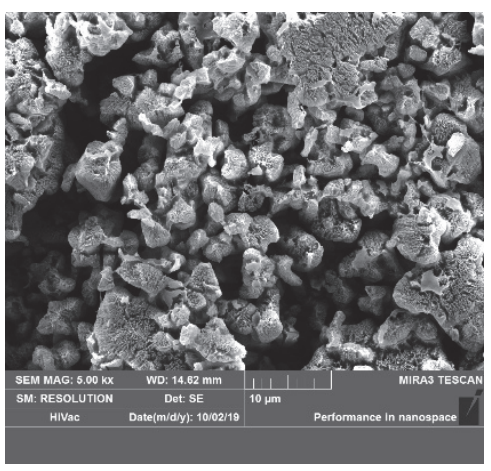

(b)

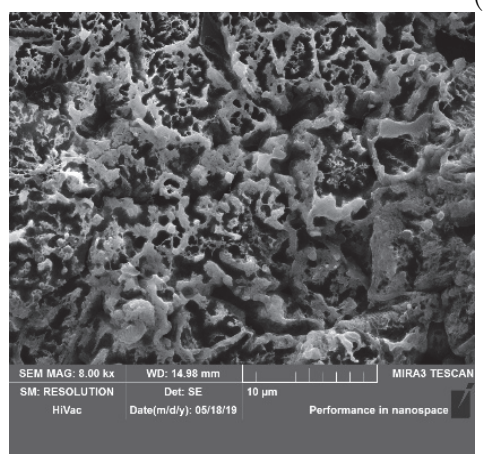

(d)

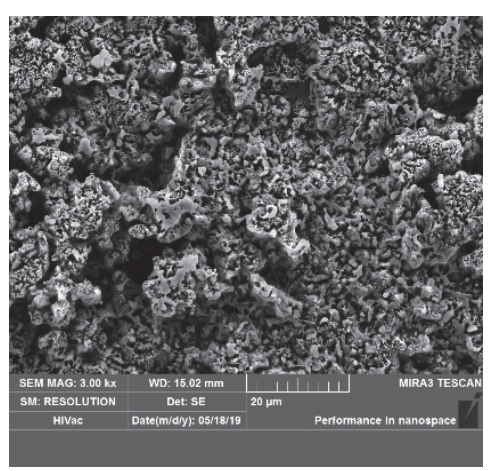

(c)

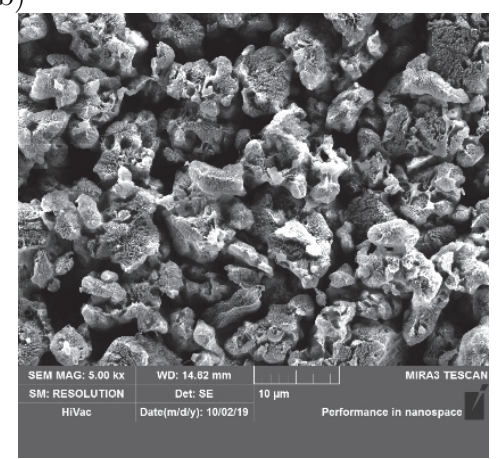

(e)

Figure 3. SEM images of different samples.

\subsection{Thermolysis of AN Samples before and after Coating}

The thermal decomposition characteristics of the raw material AN and the four coated AN samples were analyzed by DSC and the results are shown in Figure 4. Each sample in the figure is the result of testing at a heating rate of $20^{\circ} \mathrm{C} / \mathrm{min}$. It can be seen from the figure that the thermal decomposition peak of the raw 
material AN is $293.9^{\circ} \mathrm{C}$. The thermal decomposition peaks of AN after coating with PS, PMMA, PVC and $\mathrm{NC}$ are lower than the thermal decomposition peak of $\mathrm{AN}$ before coating, which are $287.6^{\circ} \mathrm{C} \mathrm{C}, 283.4^{\circ} \mathrm{C}$, $283.0^{\circ} \mathrm{C}$ and $289.5^{\circ} \mathrm{C}$, respectively. The first peak in the DSC curve of AN is the heat released by the slow redox reaction between $\mathrm{AN}$ and oxygen in the air under heating. The second peak is the edonthermic peak where AN completely melts. For the samples coated with the four binders, the endothermic peak also appeared at $100-150{ }^{\circ} \mathrm{C}$, and it was more obvious than the exothermic peak of the raw material. The binder decomposed slowly as the heating temperature increased and release heat. The second peak in the curve corresponds to the melting peak of the main explosive AN. The endothermic peak for other samples is due to the deformation of the joint before $100{ }^{\circ} \mathrm{C}$, the transition temperature increases, and the exposed $\mathrm{AN}$ Mostly, the exposed AN slowly melts within $150{ }^{\circ} \mathrm{C}$.

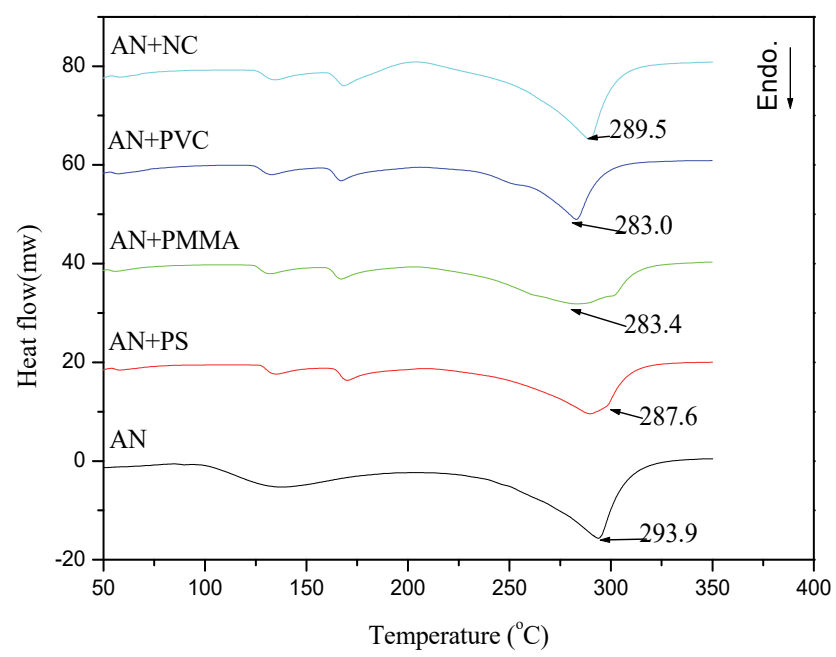

Figure 4. The result of testing at a heating rate of $20^{\circ} \mathrm{C} / \mathrm{min}$ of each sample.

The decomposition peak temperatures of AN samples before and after coating are measured at $5{ }^{\circ} \mathrm{C} / \mathrm{min}$, $10{ }^{\circ} \mathrm{C} / \mathrm{min}, 15{ }^{\circ} \mathrm{C} / \mathrm{min}$ and $20^{\circ} \mathrm{C} / \mathrm{min}$. And the test results are shown in Figure 5 below. A clear endothermic peak appears on the DSC curve, which corresponds to the thermal decomposition of the explosive, and as the heating rate increases, the temperature of the decomposition peak also increases.

According to the decomposition peak temperature and starting temperature of different samples at different heating rates, the thermodynamic and kinetic data of all samples are calculated according to formulas $(2) \sim(6){ }^{[14,15]}$. The calculation results are shown in Table 3 . The $E_{K}$ in the kinetic data is numerically similar to the $\Delta H^{\neq}$in the thermodynamic data. $\Delta H^{\neq}$characterizes the heat required for a substance from normal to transition state and its physical meaning is similar to the activation energy $E_{K}$. $\Delta G^{\neq}$is the Gibbs free energy of the change of matter from normal to transition state. Since $\Delta G^{\neq}$is positive, it indicates that the activation of the substance is not spontaneous and requires a certain amount of energy, which is numerically equal to $\Delta H^{\ddagger}$. In the table, $\Delta S^{\ddagger}$ is the absolute value data, which is a measure of the degree of chaos in the system.

$$
\begin{gathered}
\ln \frac{\beta}{T_{p}^{2}}=\ln \frac{R \cdot A_{K}}{E_{K}}-\frac{E_{K}}{R} \cdot \frac{1}{T_{p}} \\
k=A_{K} \cdot \operatorname{Exp}\left(-\frac{E_{K}}{T_{p} \cdot R}\right) \\
A \exp \left(-\frac{E_{K}}{R T_{P}}\right)=\frac{K_{B} T_{P}}{h} \exp \left(-\frac{\Delta G^{\neq}}{R T_{P}}\right) \\
\Delta H^{\neq}=E_{K}-R T_{P} \\
\Delta G^{\neq}=\Delta H^{\neq}-T_{P} \Delta S^{\neq}
\end{gathered}
$$


where $T_{P}$ is the peak temperature, $\mathrm{K} ; K_{B}$ and $h$ are the Boltzmann $\left(K_{B}=1.381 \times 10^{-23} \mathrm{~J} / \mathrm{K}\right)$ and Plank constants $\left(h=6.626 \times 10^{-34} \mathrm{~J} / \mathrm{s}\right)$, respectively; $\beta$ is the heating rate; $E_{K}$ and $A_{\mathrm{K}}$ are the activation energy and preexponential factor, respectively; $\Delta H^{\neq}$is the activation enthalpy, $\mathrm{J} / \mathrm{mol} ; \Delta G^{\neq}$is the activation free energy, $\mathrm{J} / \mathrm{mol}$; and $\Delta S^{*}$ is the activation enthalpy $\mathrm{J} /(\mathrm{mol} \cdot \mathrm{k})$.

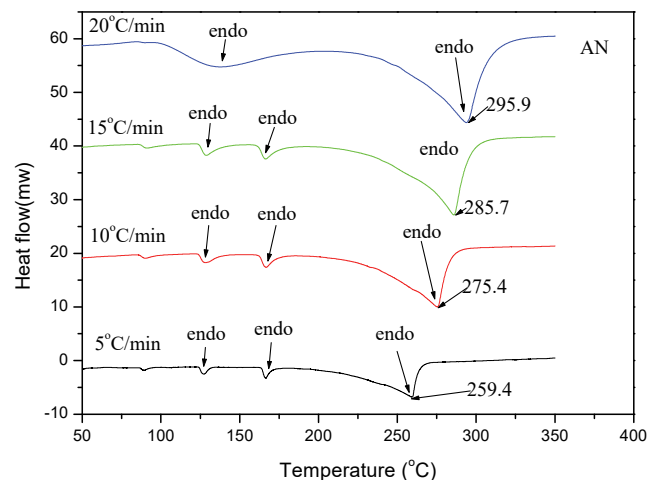

(a)

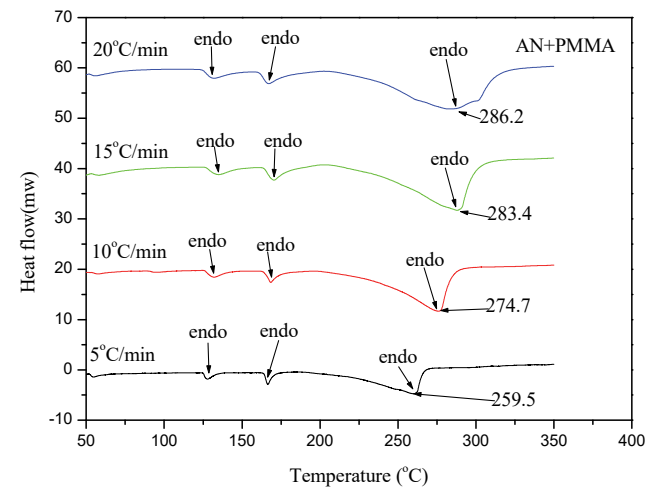

(c)

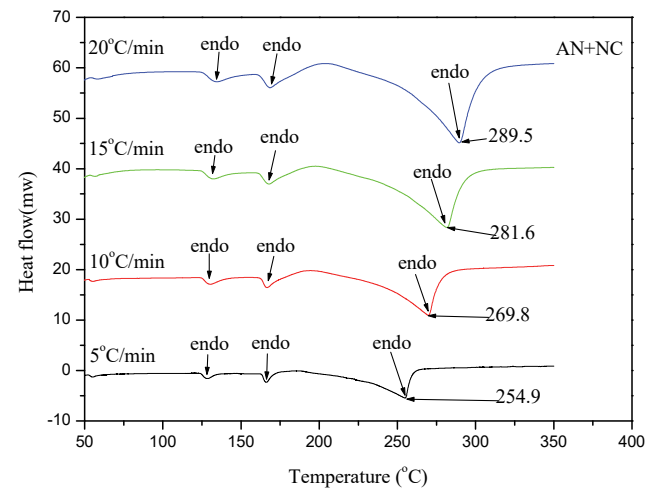

(b)

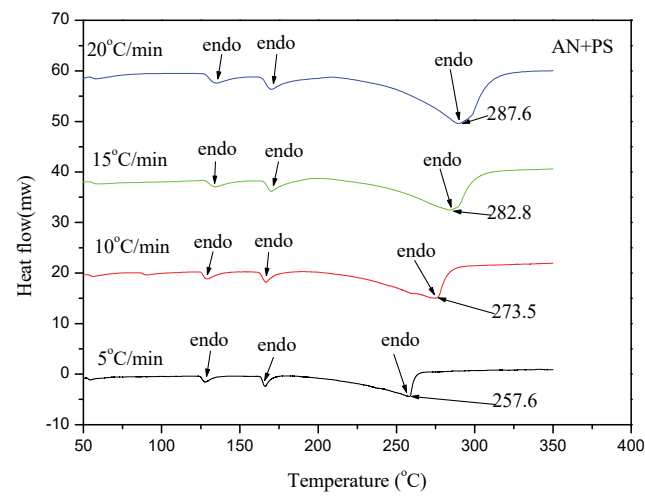

(d)

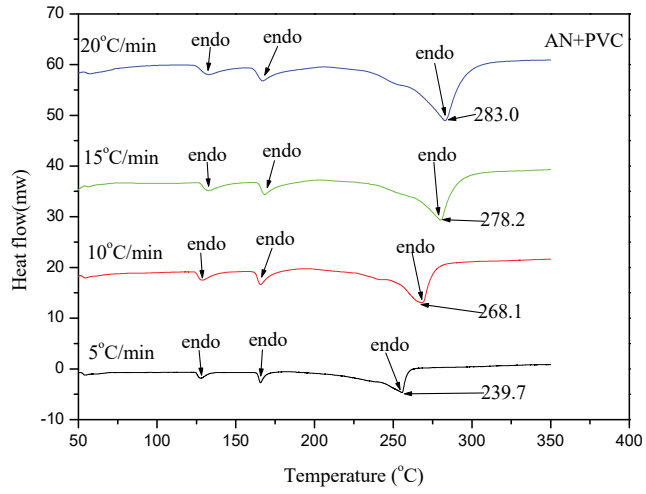

(e)

Figure 5. DSC test results of different samples

Table 3. Thermodynamic calculation results of different samples.

\begin{tabular}{llllllll}
\hline Samples & $\boldsymbol{T}_{\boldsymbol{P}} / \boldsymbol{K}$ & $\boldsymbol{\Delta} \boldsymbol{H}^{\neq} / \mathbf{k J} / \mathbf{m o l}$ & $\boldsymbol{\Delta} \boldsymbol{G}^{\neq} / \mathbf{k J} / \mathbf{m o l}$ & $\boldsymbol{\Delta} \boldsymbol{S}^{\neq} / \mathbf{J} /(\mathbf{m o l} \cdot \mathbf{k})$ & $\boldsymbol{E}_{\boldsymbol{K}} / \mathrm{kJ} / \mathbf{m o l}$ & $\boldsymbol{I n} \boldsymbol{A}_{\boldsymbol{K}}$ & $\boldsymbol{k} / \boldsymbol{s}^{\mathbf{1}}$ \\
\hline AN & 558.85 & 87.55 & 193.23 & 189.10 & 92.2 & 8.336 & 0.533 \\
AN+PMMA & 556.55 & 109.27 & 204.30 & 170.87 & 113.9 & 10.539 & 0.663 \\
AN+NC & 554.75 & 84.69 & 190.52 & 190.77 & 89.3 & 8.126 & 0.523 \\
AN+PS & 555.95 & 98.48 & 198.30 & 179.55 & 103.1 & 9.479 & 0.602 \\
AN+PVC & 551.35 & 58.52 & 172.68 & 207.05 & 63.1 & 5.560 & 0.375 \\
\hline
\end{tabular}


The results show that the apparent thermal decomposition activation energy EK of the raw material AN and the samples coated with PMMA, NC, PS, and PVC are $92.20 \mathrm{~kJ} \cdot \mathrm{mol}^{-1}, 113.90 \mathrm{~kJ} \cdot \mathrm{mol}^{-1}$, and 89.30 $\mathrm{kJ} \cdot \mathrm{mol}^{-1}, 103.10 \mathrm{~kJ} \cdot \mathrm{mol}^{-1}$ and $63.10 \mathrm{~kJ} \cdot \mathrm{mol}^{-1}$. After coating with PMMA and PS, the apparent activation energy of the samples increased, but after coating NC and PVC, the apparent activation energy decreased. For the rate constant k, PMMA and PS are coated larger than the raw material, and NC and PVC are coated smaller than the raw material. Use Equations 3 to 5 to obtain activation enthalpy $\left(\Delta H^{\ddagger}\right)$, activation entropy $\left(\Delta S^{\neq}\right)$, and activation free energy $\left(\Delta G^{\neq}\right)$. In this study, the activation enthalpy after coating PMMA, NC, and PS is greater than that of the raw material, indicating that the activation molecules heated to the transition state after coating the three coating agents require less energy than the raw material AN molecules. The activation enthalpy after coating PVC is less than that of the raw material, indicating that the activation molecule heated to the transition state after coating PVC requires more energy than the raw material AN molecule. $\Delta \mathrm{G}^{\neq}$is the change in Gibbs energy that brings the explosive molecule to the activated state. In this paper, $\Delta \mathrm{G}^{\neq}$in each sample is a positive number, which means that their change from normal to transition state cannot be carried out spontaneously and requires energy absorption.

\subsection{XRD Analysis of AN Samples before and after Coating}

XRD analysis was performed on the raw material AN sample, the uncoated AN sample after refining, and the samples coated with the four materials, and the results are shown in Figure 6. Figure 6 (a) is the XRD patterns of the raw material AN and the refined AN. And xhAN represents the refined AN. It can be seen from the figure 6(a) that the position of the AN diffraction peak is unchanged before and after refinement, indicating that the AN is refined by mechanical ball milling, and the crystal form of AN is not changed. Figure 6(b) shows the XRD patterns of AN after coating of raw material AN and different materials. It can be seen from the figure $6(\mathrm{~b})$ that the position of the diffraction peaks before and after coating are basically unchanged, indicating that the crystal form of AN is not changed after coating. However, after coating with PS, PMMA and PVC, the intensity of the diffraction peaks increase. This is because the crystal high polymer of PS, PMMA and PVC enhances the diffraction peak intensity of AN after coating. The intensity of the diffraction peak decreased with $\mathrm{AN}$ coated with $\mathrm{NC}$, indicating that $\mathrm{NC}$ is an amorphous polymer, which reduces the intensity of the diffraction peak of AN after coating.

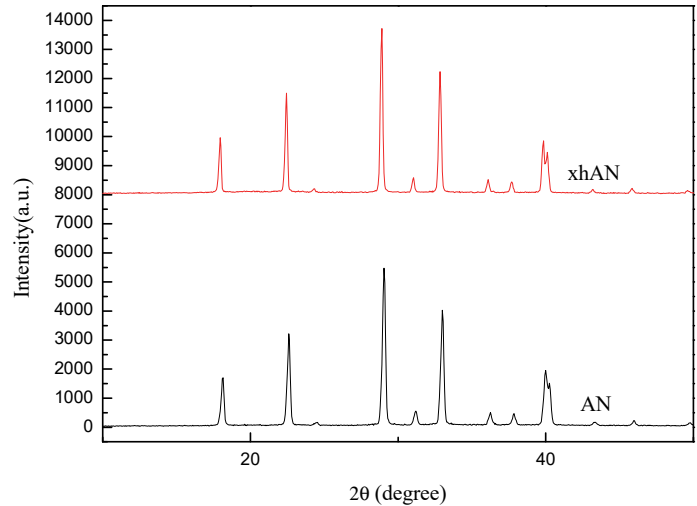

(a)

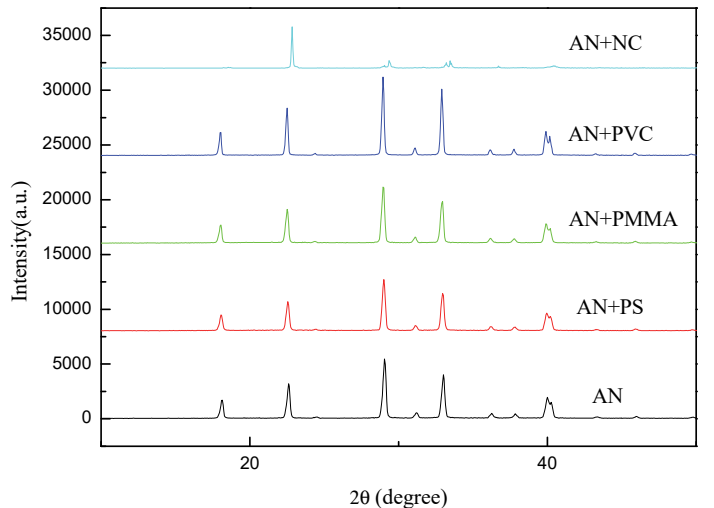

(b)

Figure 6. XRD patterns of different samples.

\subsection{IR Analysis of AN Samples before and after Coating}

In order to further verify whether the AN was effectively coated, the samples before and after the coating were subjected to IR test, and the results are shown in Figure 7. Comparing the infrared spectrum of the raw material and the sample coated with PMMA, it was found that the curved curve of the $\mathrm{C}=\mathrm{O}$ bond and the $\mathrm{C}-\mathrm{H}$ out-of-plane bending vibration occurred at the $1732.07 \mathrm{~cm}^{-1}$ and $752.13 \mathrm{~cm}^{-1}$ respectively. And this shows that PMMA is coated on the AN surface. Comparing the curves of the samples coated with PVC, 
$\mathrm{NC}$ and PS with the test curves of the raw materials, it was found that the $\mathrm{C}=\mathrm{C}$ bond stretching vibration appeared at $1644.49 \mathrm{~cm}^{-1}, 1640.60 \mathrm{~cm}^{-1}$ and $1640.96 \mathrm{~cm}^{-1}$, respectively. And the PS-coated sample also showed CH-external bending stretching vibration at $753.73 \mathrm{~cm}^{-1}$. These results show that the above three materials are also effectively coated with raw materials.

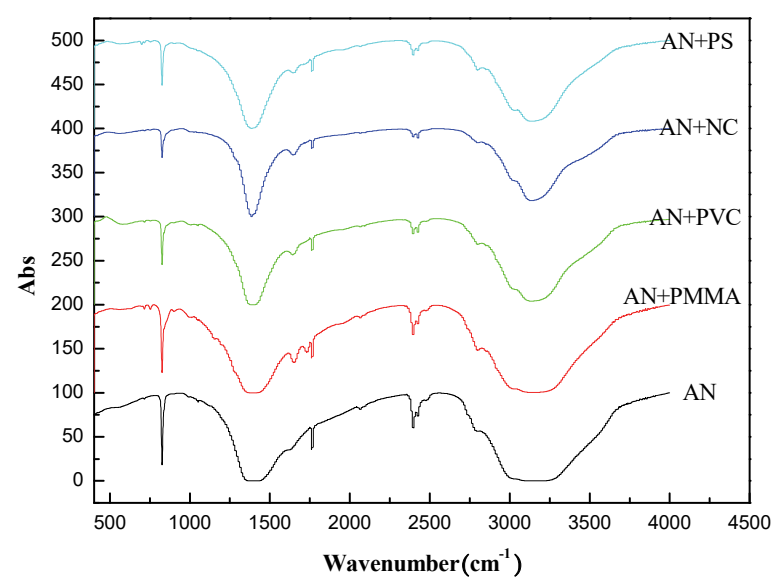

Figure 7. IR test patterns of samples before and after coating.

\subsection{Anti-hygroscopic Analysis of AN Samples before and after Coating}

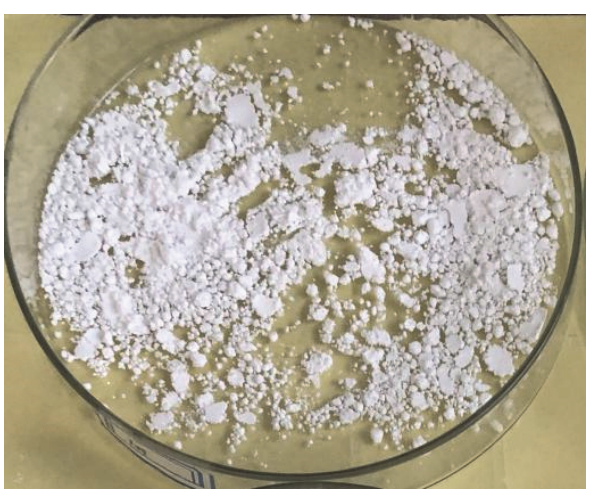

(a)

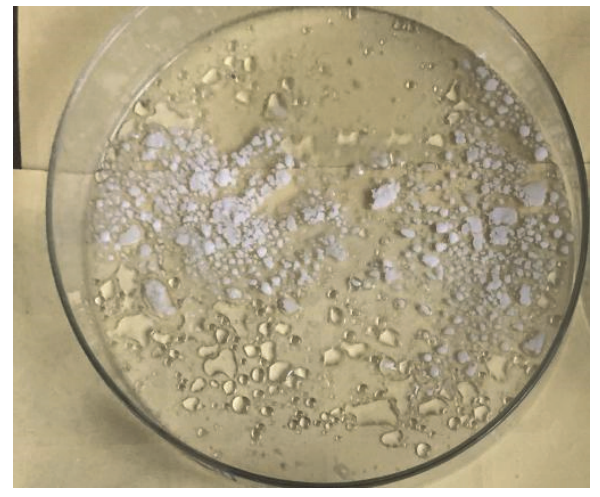

(b)

Figure 8. The raw material AN before and after moisture absorption.

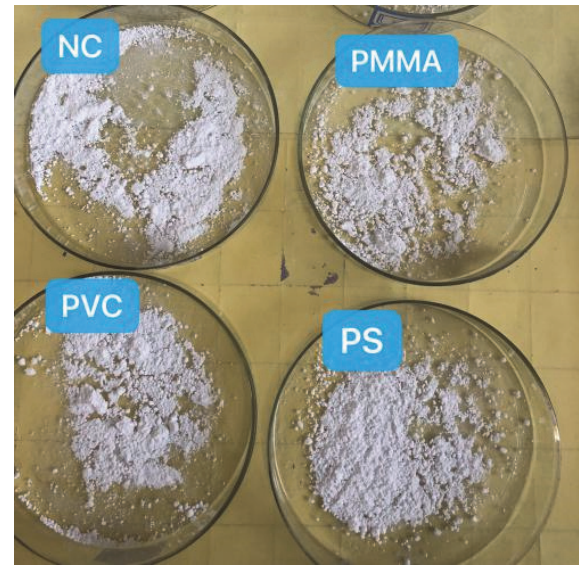

(a)

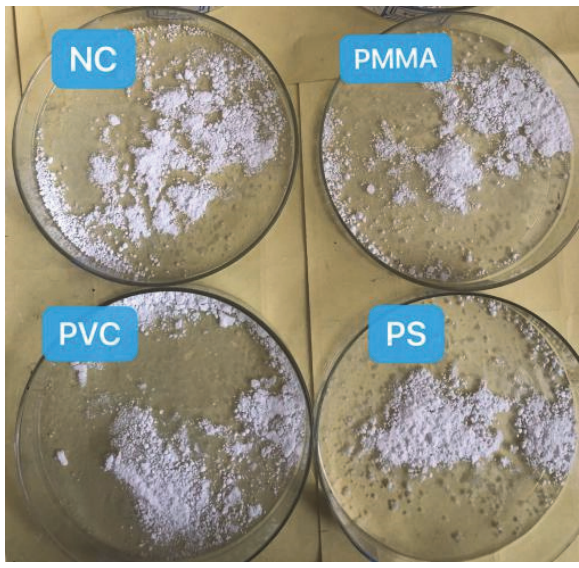

(b)

Figure 9. AN samples coated with the four materials before and after moisture absorption. 
The AN material and the four coated AN samples were taken $1( \pm 0.2) \mathrm{g}$ in a vacuum drying oven for $2 \mathrm{~h}$. Then prepare a humidity environment with a relative humidity of $75 \%$ (sulfuric acid aqueous solution with a sulfuric acid mass fraction of $30.6( \pm 0.05) \%)$. The sample was placed in an environment with a temperature of $25^{\circ} \mathrm{C}$ and a relative humidity of $75 \%$ for hygroscopicity experiments. The raw material AN before and after moisture absorption are as shown in (a) and (b) of Figure 8. The pictures of the AN samples coated with the four materials before and after moisture absorption are shown in Fig. 9 (a) and (b).

The weight of the samples are recorded every hour during the test for measuring hygroscopicity. Each sample is weighed twice each time, and the data is averaged of the two test data. The data of the first 10 hours and the 24th hour are recorded during the experiment, and the weight gain of each sample is calculated. The results are shown in Table 4 below. According to the weight gain data of different samples in Table 4, the moisture absorption rate data of the samples after standing for 24 hours in a moisture-absorbing environment with a temperature of $25{ }^{\circ} \mathrm{C}$ and a relative humidity of $75 \%$ can be calculated as shown in Table 5 below. The weight gain curves of different samples are shown in Figure 10.

The method of measuring the moisture absorption rate is to place a certain quality sample in a closed environment with a certain temperature and humidity, and weigh the sample's mass change within a certain period of time., The moisture absorption rate is the percentage of the mass gain of the sample in a certain period of time, the calculation formula is:

$$
\mathrm{RH}=\left(\mathrm{A}-\mathrm{A}_{0}\right) / \mathrm{A}_{0} \times 100 \%
$$

$\mathrm{RH}$ is the moisture absorption rate of the sample, \%. A is the quality of the sample after being placed in a hygroscopic environment for a period of time, $\mathrm{g} . \mathrm{A}_{0}$ is the mass of the sample before moisture absorption, g.

Table 4. Weight gain data of different samples.

\begin{tabular}{llllll}
\hline Hours (h) & AN & PS+AN & PMMA+AN & PVC+AN & NC+AN \\
\hline 0 & 1.0265 & 1.0128 & 0.9912 & 1.0134 & 1.0193 \\
1 & 1.0267 & 1.0132 & 0.9927 & 1.0139 & 1.0194 \\
2 & 1.0270 & 1.0133 & 0.9930 & 1.0140 & 1.0195 \\
3 & 1.0295 & 1.0134 & 0.9931 & 1.0146 & 1.0197 \\
4 & 1.0332 & 1.0137 & 0.9934 & 1.0147 & 1.0198 \\
5 & 1.0397 & 1.0138 & 0.9937 & 1.0148 & 1.0200 \\
6 & 1.0446 & 1.0139 & 0.9938 & 1.0152 & 1.0230 \\
7 & 1.0502 & 1.0144 & 0.9944 & 1.0165 & 1.0254 \\
8 & 1.0563 & 1.0146 & 0.9982 & 1.0228 & 1.0276 \\
9 & 1.0598 & 1.0156 & 1.0027 & 1.0280 & 1.0297 \\
10 & 1.0651 & 1.0173 & 1.0090 & 1.0328 & 1.0326 \\
24 & 1.1642 & 1.1335 & 1.0956 & 1.0869 & 1.0918 \\
\hline
\end{tabular}

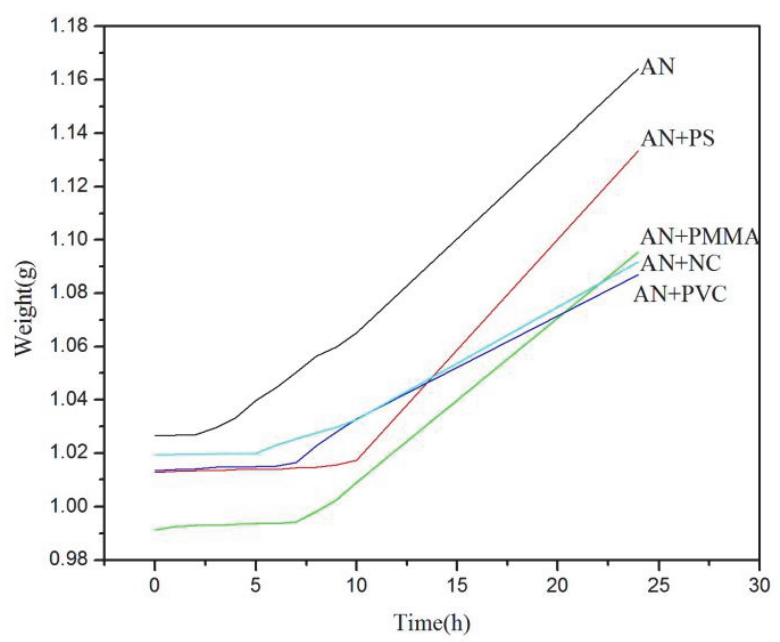

Figure 10. Line chart of sample weight gain 
Table 5. The moisture absorption rate data of the samples.

\begin{tabular}{cc}
\hline Samples & Moisture Absorption Rate $/ \%$ \\
\hline AN & 13.41 \\
PS+AN & 11.92 \\
PMMA+AN & 10.53 \\
PVC+AN & 7.25 \\
NC+AN & 7.12 \\
\hline
\end{tabular}

According to the moisture absorption rate data of the sample in Table 5, the moisture absorption rates of the raw material AN and the samples coated with PS, PMMA, PVC and NC were $13.41 \%, 11.92 \%$, $10.53 \%, 7.25 \%$ and $7.12 \%$, respectively. The AN coated with PS, the AN after PMMA coating, the AN after PVC coating, and the AN after $\mathrm{NC}$ coating have a relative moisture absorption rate compared with the raw material before coating and they are: $88.89 \%, 78.52 \%, 54.06 \%$ and $53.09 \%$. Therefore, the AN moisture absorption rate after coating with PS, PMMA, PVC, and NC decreased by $11.11 \%, 21.48 \%$, $45.94 \%$, and $46.91 \%$, respectively, compared with the moisture absorption rate of the raw material AN. It can be found that the four coating materials have the best anti-hygroscopic effect on AN compared with $\mathrm{NC}$ coating AN, followed by PVC coating material, then PMMA coating material, and the worst effect is to use PS as coating material. The calculation result of the binding energy characterizes the stability of the combination of the coating agent and the explosive. The final moisture absorption rate does not correspond to the calculation result of the binding energy, because the hydrophobicity of the coating agent itself also affects the moisture absorption rate. Among the four coating agents, the worst hydrophobicity is PS, and the best hydrophobicity is PMMA, so the order of the calculation results of the moisture absorption rate and the calculation results of the binding energy is not completely consistent.

\section{Conclusions}

Herein, the combined energy of PS, PMMA, PVC and NC and AN calculated by Materials Studio software are: $-22.32 \mathrm{kcal} \cdot \mathrm{mol}^{-1},-53.38 \mathrm{kcal} \cdot \mathrm{mol}^{-1},-42.15 \mathrm{kcal} \cdot \mathrm{mol}^{-1}$ and -34.12 , respectively. Kcal $\cdot \mathrm{mol}^{-1}$. The order of the absolute value of the binding energy is: $\mathrm{PMMA}>\mathrm{PVC}>\mathrm{NC}>\mathrm{PS}$, indicating that the order of interaction between the additive and the crystal face is: $\mathrm{PMMA}>\mathrm{PVC}>\mathrm{NC}>\mathrm{PS}$.

On the other hand, the characterization tests of the AN samples before and after coating show that the refined AN particle diameter obtained by the mechanical ball milling method is within $5 \mu \mathrm{m}$ and is ellipsoidal. Moreover, the four coating materials are effectively coated on the AN. The crystal structure of the AN is not changed before and after coating.

In terms of improving the hygroscopicity of $\mathrm{AN}$, the improvement effect of the four coating materials is $\mathrm{NC}>\mathrm{PVC}>\mathrm{PMMA}>\mathrm{PS}$. Compared with the pre-coated raw materials, the AN coated with PS, PMMA, PVC and NC had relative moisture absorption rates of $88.89 \%, 78.52 \%, 54.06 \%$ and $53.09 \%$, respectively. The AN moisture absorption rate after coating with PS, PMMA, PVC, and NC decreased by $11.11 \%$, $21.48 \%, 45.94 \%$, and $46.91 \%$, respectively, compared with the moisture absorption rate of the raw material AN. The density of the four materials and the stability to water are all $\mathrm{NC}>\mathrm{PVC}>\mathrm{PMMA}>\mathrm{PS}$, so these are also the reasons for the difference in the improvement of AN hygroscopicity between the four materials.

Acknowledgments. This research was supported by the National Natural Science Foundation of China (Grant No.: 51676082).

Conflicts of Interest: The authors declare no conflict of interest.

\section{References}

1. D. Trache, T. M Klaptke, L Maiz, "Recent advances in new oxidizers for solid rocket propulsion," Green Chemistry, vol. 19, pp. 4711-4736, 2017.

2. J. Zhang, R. Yang, Y. Liu, "Study on the Hygroscopicity of Ammonium Nitrate," Chinese Journal of Explosives, vol. 3, pp. 22-25, 2001. 
3. S. Cheng, Z. Ma, Z. He, "Study on hygroscopicity and stability of coated ammonium nitrate propellant," Chemical Propellants \& Polymeric Materials, vol. 1, pp. 90-92, 2014.

4. A. Yang, N. Luo, S. Zheng, "Summary of Research on Ammonium Nitrate Modification at Home and Abroad," Coal Mine Blasting, vol. 10, pp. 21-23, 2008.

5. Z. Wang, Z. He, Z. Ma, "Study on the hygroscopicity of modular ammonium nitrate propellant," New Chemical Materials,vol. 3, pp. 116-120, 2016.

6. C. An, X. Song, Y. Wang, "Research progress on surface coating of ammonium nitrate explosives," Chinese Journal of Energetic Materials, vol. 15, pp. 188-192, 2007.

7. J. Chen, X. Wang, H. Xu, "Surface modification of ammonium dinitramide (ADN)," ADN Synthetic and Applied Symposium Proceedings. Beijing, 2006, pp. 116-120.

8. Y. Wang, X. Song, C. Liu, "Basic properties of energy-containing plasticizer diglycerol tetranitrate," Solid Rocket Technology, vol. 2, pp. 198-204, 2019.

9. X. Cheng, S. Wang, H. Ba, "Study on the improvement of hygroscopicity by polystyrene coated with ammonium nitrate," Adhesive academic paper research report and monograph. Shanghai, 2013, pp. 76-78.

10. H. Liu, H. Deng, S. Deng, "Study on the Synthesis of Polymethyl Methacrylate," Chemical Engineering 86 Equipment, vol. 5, pp. 1-2, 2018.

11. J. Yu, S. Wang, "Study on the Adsorption Mechanism of Nitrocellulose on Creatinine," Chinese Journal of Organic Chemistry, vol. 3, pp. 1033-1035, 2003.

12. V. Gettwer, A. Franzin, M. A. Bohn, "Ammonium dinitramide/glycidyl azide polymer(ADN/GAP) composite propellants with ADN without metallic fuels," International Journal of Energetic Material and Chemical Propulsion, vol. 1, pp. 61-79, 2017.

13. A. Rahman, J. Chin, F. Kabir, "Characterzation and trust measurements from electrolytic decomposition of ammonium dinitramide (ADN) based liquid monopropellant FLP-103 in MEMS thruster," Chinese Journal of Chemical Engineering, vol. 26, pp. 1992-2002, 2018.

14. H. E. Kissinger, "Reaction kinetics in different thermal analysis," Analytical Chemistry, vol. 29, pp. 1702-1706, 1957.

15. M. R. Sovizi, S. S. Hajimirsadeghi, B. Naderizadeh, "Effect of particle size on thermal decomposition of nitrocellulose," Journal of Hazardous Materials, vol. 168, pp. 1134-1139, 2009.

16. Y. Nishiyama, P. Langan, M. Wada, "Looking at hydrogen boods in cellulose," Acta Crystallographica, vol. 66, pp. 1172-1177, 2010.

17. K. Zhang, O. Ni, Z. Yu, "The effect of potassium nitrate on inhibiting the phase transition of ammonium nitrate ANIV-ANIII and its application in powdered emulsified explosives," Blasting Equipment, vol. 41, pp. 4-7, 2012.

18. L. Zhang, "CL-20 explosive phase change and chemical reaction molecular dynamics calculation," Doctoral Dissertation, Beijing Institute of Technology, 2016.

19. Z. Chen, X. Wang, R. Gou, "Molecular dynamics simulation of the interfacial interaction between CBNT and polymer binders," Journal of Explosives 83 Propellants, vol. 5, pp. 479-483, 2018.

20. W. Tian, "Discussion on the operation mechanism of planetary ball mill in laboratory," Enterprise Technology Development, vol. 36, pp. 80-81, 2017.

21. J. Song, X. Guo, F. Li, "Preparation and characterization of ultrafine spherical ammonium perchlorate," Solid State Rocket Technology, vol. 37, pp. 521-524, 2014.

22. S. Zhao, X. Song, Y. Wang, "Preparation and characterization of nano-TAGN by mechanical ball milling," Initiators \&3 Pyrotechnics, vol. 13, pp. 22-25, 2017.

23. C. An, P. Ding, B. Ye, "Carbon-coated copper nanoparticles prepared by detonation method and their thermocatalysis on ammonium perchlorate," AIP Adv. Vol. 7, pp. 035324, 2017.

24. J. Tao, X. Wang, X. Zhao, "Simulation of binding energy and mechanical properties of $\varepsilon$-CL-20/energetic bonding compound system," Journal of Energetic Materials, vol. 23, pp. 315-322, 2015.

25. C. An, "Surface coating of nitramine explosives and its effect on propellant properties," Doctoral Dissertation, Nanjing University of Science and Technology, 2008.

26. N. Li, L. Xiao, X. Yan, "Study on the coating of RDX with GAP-based energetic polyurethane elastomer," Solid State Rocket Technology, vol.35, pp. 212-215, 2012.

27. Y. Wang, X. Song, D. Song, "Synthesis, thermolysis, and sensitivities of HMX/NC energetic nanocomposites," Hazardous Materials, vol. 28, pp. 73-83, 2016.

28. B. Ye, C. An, Y. Zhang, "One-step ball milling preparation of nanoscale CL-20/graphene oxide for significantly reduced particle size and sensitivity," Nanoscale Research Letters, vol. 13, pp. 38-42, 2018.

29. X. Xu, X. Xiao, H. Huang, "Molecular dynamics simulation of structure and properties of $\varepsilon$-CL-20 based 
PBX_— preparation of HEDM theory formula design," Science in China, Chemistry, vol. 37, pp. 556-563, 2007. 30. X. Guo, H. Nan, X. Qi, "RDX and HMX crystals mechanical properties and molecular dynamics simulations in response to impact loads," Energetic Materials, vol. 14, pp. 485-489, 2013.

31. D. Zhang, C. Zheng, S. Heng, "The thermal decomposition kinetics compensation effect of RDX high energy nitramine propellant," Chinese Journal of Explosives, vol.33, pp. 82-85, 2014. 